\title{
Mathematical modeling of allergenic pollen propagation in atmospheric layer
}

\author{
Medveditsyna Olga Sergeevna \\ dept.physics and medical informatics \\ Kirov State Medical University \\ Kirov City, Russian Federation \\ ossitnikova@yandex.ru, 0000-0002- \\ 4557-6623
}

\author{
Rychkov Sergey Leonidovich \\ research and educations center of \\ biotecnology \\ Vyatka State University \\ Kirov City, Russian Federation \\ rychkov@list.ru 0000-0002-7592-617X
}

\author{
Shatrov Anatoly Victorovich \\ dept.of automatics and computing \\ Vyatka State University, dept.physics \\ and medical informatics Kirov State \\ Medical University \\ Kirov City, Russian Federation \\ avshatrov1@yandex.ru 0000-0002- \\ $5295-571 X$
}

\begin{abstract}
The quasi-two-dimensional model of impurity propagation early designed elsewhere is modified for transporting of allergenic plant pollen from spread forested areas in vicinity of a large city. The model includes consideration of mesoscale hydrothermodynamical processes in the lower atmosphere taking into account thermal nonuniformities of the underlying surface in the urban and suburban environs. The boundary conditions and the model coefficients are determined using the parametrization method. Some results of numerical calculations are presented. The calculations were performed using parallelized algorithms on the cluster supercomputer of the Vyatka State University. They show that, due to the action of an inhomogeneous horizontal temperature gradient in the lower atmosphere, vortex flows can be formed above populated areas.
\end{abstract}

Keywords - allergenic pollen, mathematical modeling, impurity propagation, high-performance calculations

\section{Introduction}

Pollen grains contained in the atmosphere have the ability to cause allergic diseases. An increase in the number of pollen allergy diseases in the second half of the 20th century led to growth of interest in the atmospheric transfer of pollen around the world. 2018 is called by World Health Organization (WHO) to be the year of the allergy pandemic. The prevalence (30-60\% of the population) of allergic diseases (every third inhabitant of the planet suffers from allergic rhinitis and every tenth of bronchial asthma) turned allergies into a global medical and social problem. The percentage of people suffering from allergies (mainly among the young people) is significantly higher in highly developed countries than in developing ones. The problem of allergic diseases spreading among children is very acute. Moreover, there are much fewer allergic among the villagers, while in Moscow every third one suffers from hay fever, in Berlin every fourth, in New York - every sixth. The reason is that allergies are caused not so much by the plants themselves as their pollen, which absorbs all the harmful emissions and polluting particles that are presented in catastrophic amounts in the air of the metropolis. A sharp increase in the number of allergic diseases (AD) occurs in April-May in conditions of central Russia, when the flowering of alder, birch, willow, maple and poplar begins. During this period, pollen calendars are being formed for most large cities, patterns of pollen content of certain plant species in the atmosphere are investigated, the influence of meteorological factors is determined, a network of permanently operating stations for monitoring pollen is created [1]. The well-known information search engine Yandex publishes daily a map of distributions of pollen emissions of various origin in vicinities of large cities. Yandex.Pogoda forms a special map for those persons who are allergic to pollen.

\section{Morphology of a pollen allergy}

However, these distributions are not confirmed with numerical parameters and can be considered as illustrative material of possible occurrences of aerosol pollen components. An important factor in assessing the degree of pollution by aerosol pollen is the knowledge of the physical parameters during the pollen transfer. Data for the mass proportion of grains of various tree species and the procedure of measuring them are given in [2]. Table I shows the data for the distribution of masses of grains and the corresponding proportion of the main tree species for the European part of Russia.

TABLE I. DISTRIBUTION OF POLLEN GRAINS ON MASS

\begin{tabular}{|l|l|l|}
\hline Wood species & Mass of grains & $\begin{array}{l}\text { Percentage in } \\
\text { distribution }\end{array}$ \\
\hline Fir & 82,4 & 0,06 \\
\hline Spruce & 63,1 & 0,24 \\
\hline Pine & 15,5 & 0,18 \\
\hline Linden, oak, maple, & 10,7 & \\
\hline elm & 4 & 0,02 \\
\hline Aspen & 3,9 & 0,1 \\
\hline Birch tree & 3,5 & 0,26 \\
\hline Alder & 3,5 & 0,09 \\
\hline Poplar & 2,5 & 0,03 \\
\hline Willow & & 0,02 \\
\hline
\end{tabular}

One can see that the presented data are grouped by grain weight in three main groups: heavy (spruce, fir and pine), medium (linden, oak, maple, elm) and light (aspen, birch, alder, poplar, willow). Taking into account the presented data, attention should be paid to the fact that the mass of allergenic pollen grains has the greatest influence on the transfer processes and there is a group of light fractions. It is the last group that represents the most dangerous impact on the development of AD in large cities. 


\section{Math Model and Simulation of Problem}

The statement of the problem of the transfer of pollutants in the form of aerosol components involves the use of a sequence of mathematical models built on the principle "from simple to complex." This sequence is determined by the choice of modeling scale. If we define a certain, rather small neighborhood of the impurity distribution region over a finite period of time, then it is quite reasonable to use simplified (including stationary) models allowing exact solutions. But it is necessary to take into account the complex dynamics of the velocity and temperature fields, surface inhomogeneity and boundary conditions in the case of problems with extended geometry or with sufficiently powerful pollutant sources, for example, when estimating emissions from large industrial enterprises. In [3], [4], mesoscale models of the bioaerosol components transport are considered both for individual types of plant pollen [5] and in the aggregate of inert impurities [6]. The equations of momentum, temperature, impurities and moisture transfer are used for calculations [7]. In [6] the mesoscale model of the surface layer is used to calculate transfer of fungal mold and estimate its interaction with atmospheric flow moisture. This work presents a mesoscale quasi-two-dimensional model of the transfer of pollen grains of various fractions, which are the most common types of plant aerosol allergens: birch, alder, poplar, maple, willow on a micro- and mesoscale [8]. A finite-difference explicit calculation method was used for numerical implementation. The construction of a parallel version of the calculation algorithm was based on the principle of geometric decomposition of the grid domain.

\section{Results of Modeling}

The parallel computational algorithm was implemented in the Fortran version of Intel Cluster Studio for Linux Open MP, installed on the Vyatka State University HPC Enigma X000 cluster supercomputer. The calculations were carried out on the basis of the system of equations with the initial and boundary conditions. The explicit difference scheme $[3,4,7]$ was used on a $1000 \times 1000$ grid. In accordance with the theory of Monin and Obukhov [8], the coefficients of vertical and horizontal turbulent viscosity, thermal conductivity, and diffusion for mesoscale turbulent processes in the lower atmosphere were assumed being the same, namely, $k_{m}, T, S=l D^{2} \quad, \quad D=400 m$ and $A_{m}, T, S=400 \mathrm{~m}^{2} / \mathrm{s}$. The wind velocity $c_{g}$ was varied from 1 to $10 \mathrm{~m} / \mathrm{s}$. In most of calculations the velocity was 2$5 \mathrm{~m} / \mathrm{s}$ in this case, the temperature inhomogeneity effect on the wind ow in the vicinity of a heat source is most clearly expressed. The interaction between aerosol impurity and the underlying surface was taken into account on the basis of the information on the nonuniformities of the temperature and absorption coefficient distributions taken from the map of land utilization of the computation domain. The air temperature $T_{S}$ varied from $18^{\circ} \mathrm{C}$ outside populated areas to $23^{\circ} \mathrm{C}$ in the city of Kirov. A minimum temperature was observable at the north boundary of the area. The coefficient of impurity absorption by the underlying surface was taken to be $\alpha=0.0139 \mathrm{~m}^{-1}$ outside populated areas and $\alpha=0.00139 m^{-1}$ on their territories. Spread impurity sources were located on the underlying surface, on the center of the region under consideration (in vicinity of the city territory).
In the calculations it was also taken that intensity of plant pollen sources are about $10^{-2} \mathrm{~kg} / \mathrm{m}^{2}$. Distribution of contaminations by the wind from north-west on Fig.1 is presented.

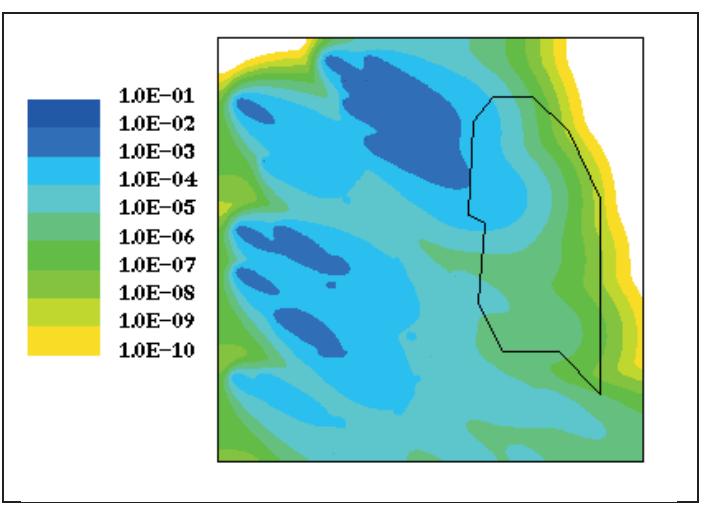

Fig. 1. Distribution of concentration of pollen for "light group" (aspen, birch, alder, poplar, willow) by the wind from north-west

\section{REFERENCES}

[1] Allergophone. URL: https://allergotop.com/ (date of coll 20.10.2019)

[2] V.V. Golovko, P.K. Kutsenogiy, E.I. Kirov, V.L. Istomin, V.A. Ryzhakov "Pollen components of atmospheric aerosols the neighborhood of Novosibirsk" [In Russian], // Optic of Atmosphere and Ocean, No 6, 1998, pp. 645-649

[3] A.V. Shatrov, K.G. Shvarts "Numerical Modeling of Mesoscale Atmospheric Impurity Transport Processes in the Environs of the City of Kirov"// Fluid Dynamics. Vol. 46, No 2. 2011, pp. 333-340. DOI: 10.1134/S0015462811020165

[4] K.G. Shvarts, V.A. Shklyaev "Mathematical Modeling of Mesoscale and Largescale Processes of Transport in Baroclinic Atmosphere" [in Russian]// Moscow-Izhevsk: Institute of Computing Research, 2015.

[5] A.V. Starchenko "Modelling of transfer of aerosol impurity in the city scale" // II-th International conference "Climatology and Glatiology of Siberia", Tomsk, 20-23, october 2015.

[6] K.G. Shvarts, Yu.A. Shvarts and V.A. Shklyaev "The twodimensional model of mesoscale processes in the lower atmosphere with allowance for inhomogeneity of temperature and air humidity."// Vycisl. meh. splos. Sred - Computational Continuum Mechanics, vol. 8, no. 1, 2015, pp. 5-15 DOI: https://doi.org/10.7242/1999$6691 / 2012.5 .3 .32$

[7] T.V. Naumovich, S.L. Rychkov, A.V. Shatrov, and K.G. Shvarts, "Software Complex for Modeling the Biotechnological Impurity Transport in the Ground Layer in the City of Kirov"// Proc. of the IV All-Russian Sci. Conf. on Math. Modeling of the Developing Economics and Ecology EKOMOD-2009 [in Russian], Vyatka Univ. Press, Kirov, 2009, pp.- 256-270.

[8] A.S. Monin, Theoretical Fundamentals of Geophysical Hydrodynamics [in Russian], Gidrometeoizdat, Leningrad 1988. 